\section{NIH overhauls psychiatric trial policy}

Target validation and experimental therapeutics trump traditional efficacy trials, says the NIMH.

The lowdown: In response to a drying pipeline and repeated failures in mental health trials, the US National Institute of Mental Health (NIMH) is revamping its clinical trial strategy. "Future trials will follow an experimental medicine approach in which interventions serve not only as potential treatments, but as probes to generate information about the mechanisms underlying a disorder," wrote NIMH Director Thomas Insel when he announced the changes. "Trial proposals will need to identify a target or mediator; a positive result will require not only that an intervention ameliorated a symptom, but that it had a demonstrable effect on a target, such as a neural pathway implicated in the disorder or a key cognitive operation." Whereas trials were previously built around establishing only the efficacy of a specific intervention, with this new strategy the NIMH hopes to gain greater insight into the pathophysiology of disease.

"The companies that have stayed in this area realize they need a more rigorous approach," Insel told Nature Reviews Drug Discovery. "That is the same process we are implementing at NIMH."

In 2012, the NIMH funded over 250 trials, at a cost of around US $\$ 100$ million. But because the new policy will add the costs of mechanistic studies to already strained budgets, the NIMH may support fewer trials in future years than it did in the past.

"The little response I have heard to date has been positive," says Insel. But, he adds, there are concerns about potential loss of serendipitous and exploratory discoveries. "Critics of our new policy might justifiably be concerned that we will miss new opportunities. At NIMH we want to be flexible and responsive to whatever delivers the most value for those with mental illness."

\section{Pharma firms pool and share cancer trial data}

Project Data Sphere aims to provide access to 25,000 patients' worth of comparator arm data from Phase III trials by the end of the year.

The lowdown: Clinical trial data sets can hold hidden gems of insight into disease and trial design. By pooling the results from comparator arms of trials of anticancer drugs into the free broad-access Project Data Sphere portal, once-rivals now hope to increase the odds of finding these gems. "There are a whole lot of things you can do with these data," says Charles Hugh-Jones, Chief Medical Officer in North America of Sanofi and one of the organizers of the new initiative.

In the short term, Hugh-Jones hopes the shared data will be used to establish baseline rates of safety events, to build natural history disease models and to optimize data collection and trial standardization. In the longer term, it may also be useful as a virtual control arm and for much more. (For a longer interview with Hugh-Jones, see Nature Rev. Drug Discov. 12, 900; 2013.)
To increase the likelihood of gaining benefits, Project Data Sphere is open and free to anyone who wants to register. It will also run a series of competitions to encourage participants to dabble with the data. A first competition will focus on using the data to better understand prostate cancer.

At launch, AstraZeneca, Bayer, Celgene, Janssen, the Memorial Sloan Kettering Cancer Center, Pfizer and Sanofi had pooled data from 3,500 patients in 9 Phase III trials. Discussions are underway with other organizations including the Alliance for Clinical Trials in Oncology, Amgen and Quintiles — and the initiative aims to add results from a further 25 trials, bringing the data set up to 25,000 patients, by the end of the year.

A few organizations have set up similar clinical trial data-sharing projects in other therapeutic areas. These have, however, been primarily for therapeutic areas that have been battered by failure, like C-Path's Online Data Repository (CODR) for Alzheimer's disease (Nature Rev. Drug Discov. 9, 499; 2010). The CODR results were central to the development of a trial simulation tool that both the US Food and Drug Administration and the European Medicines Agency supported last year for designing Alzheimer's disease trials.

\section{CDK inhibitors speed ahead}

Pfizer has posted mixed results for its palbociclib, leaving hope for trailing competitors like Novartis and Lilly. The lowdown: Cyclin-dependent kinases (CDKs), which drive the cell cycle and therefore cell proliferation, have been a focus for drug developers since the 1990s (Nature Rev. Drug Discov. 11, 892-894; 2012). With recent mixed results from Pfizer and Amgen's first-in-class CDK4 and CDK6 inhibitor palbociclib, however, they could at last be approaching the market.

Pfizer and Amgen reported results from an open-label 165-patient Phase I/II trial of palbociclib in postmenopausal women with oestrogen receptor-positive $\left(E R^{+}\right)$, human epidermal growth factor receptor 2 negative (HER2-) advanced metastatic breast cancer at the American Association for Cancer Research (AACR) annual meeting. The drug in combination with the aromatase inhibitor letrozole hit its primary end point, inducing a progression-free survival (PFS) of 20.2 months versus 10.2 months in controls. But, this improvement was down from interim analysis PFS estimates. The trial also failed on its secondary end point of overall survival: there was no statistical difference between the 37.5-month overall survival on treatment compared with the 33.3 months on control.

On the basis of these results Pfizer is reportedly considering filing for approval of palbociclib, which has breakthrough therapy designation from the US Food and Drug Administration. Two pivotal trials of the drug in combination with letrozole and with selective ER downregulator fulvestrant are also ongoing. According to Thomson Reuters Cortellis, the consensus annual sales forecasts from five analysts for the drug currently sit at US\$1.9 billion by 2019 .

A pipeline of other CDK inhibitors is also in the clinic for a range of oncology indications. Novartis initiated a Phase III trial of its CDK4 and CDK6 inhibitor LEE011 in breast cancer in January. Lilly's CDK4 and CDK6 inhibitor bemaciclib (LY2835219) is in a Phase II trial for mantle cell lymphoma, and the company also presented promising results for the drug in breast cancer at the AACR. Merck \& Co.'s dinaciclib, a CDK2, CDK2, CDK5 and CDK9 inhibitor, is in Phase II trials for acute lymphoblastic leukaemia, acute myeloid leukaemia, breast cancer, melanoma and non-small-cell lung carcinoma. 